\title{
Imprinting of the COPD airway epithelium for dedifferentiation and mesenchymal transition
}

\author{
Sophie T. Gohy ${ }^{1,2}$, Cloé Hupin ${ }^{1}$, Chantal Fregimilicka³ ${ }^{3}$ Bruno R. Detry ${ }^{1}$, \\ Caroline Bouzin ${ }^{3}$, Héloïse Gaide Chevronay ${ }^{4}$, Marylène Lecocq ${ }^{1}$, \\ Birgit Weynand $^{5}$, Maha Z. Ladjemi $^{1,6}$, Christophe E. Pierreux $^{4}$, \\ Philippe Birembaut ${ }^{7}$, Myriam Polette $^{7}$ and Charles Pilette ${ }^{1,2,6}$
}

\begin{abstract}
Affiliations: ${ }^{1}$ Université Catholique de Louvain (UCL), Institute of Experimental \& Clinical Research - Pole of Pneumology, ENT and Dermatology, Brussels, Belgium. ${ }^{2}$ Department of Pneumology, Cliniques universitaires St-Luc, Brussels, Belgium. ${ }^{3}$ Université Catholique de Louvain (UCL), Institute of Experimental \& Clinical Research - IREC Imaging Platform, Brussels, Belgium. ${ }^{4}$ Université Catholique de Louvain (UCL), CELL Unit, de Duve Institute, Brussels, Belgium. ${ }^{5}$ Department of Pathology, CHU Godinne, Yvoir, Belgium. ${ }^{6}$ Institute for Walloon Excellence in Lifesciences and Biotechnology (WELBIO), Brussels, Belgium. ${ }^{7}$ INSERM UMR-S 903, SFR CAP-Santé, University of Reims-Champagne-Ardenne, Reims, France.
\end{abstract}

Correspondence: Charles Pilette, Avenue Hippocrate 10, 1200, Brussels, Belgium.

E-mail: charles.piletteduclouvain.be

ABSTRACT In chronic obstructive pulmonary disease (COPD), epithelial changes and subepithelial fibrosis are salient features in conducting airways. Epithelial-to-mesenchymal transition (EMT) has been recently suggested in COPD, but the mechanisms and relationship to peribronchial fibrosis remain unclear. We hypothesised that de-differentiation of the COPD respiratory epithelium through EMT could participate in airway fibrosis and thereby, in airway obstruction.

Surgical lung tissue and primary broncho-epithelial cultures (in air-liquid interface (ALI)) from 104 patients were assessed for EMT markers. Cell cultures were also assayed for mesenchymal features and for the role of transforming growth factor (TGF)- $\beta 1$.

The bronchial epithelium from COPD patients showed increased vimentin and decreased ZO-1 and E-cadherin expression. Increased vimentin expression correlated with basement membrane thickening and airflow limitation. ALI broncho-epithelial cells from COPD patients also displayed EMT phenotype in up to 2 weeks of culture, were more spindle shaped and released more fibronectin. Targeting TGF- $\beta 1$ during ALI differentiation prevented vimentin induction and fibronectin release.

In COPD, the airway epithelium displays features of de-differentiation towards mesenchymal cells, which correlate with peribronchial fibrosis and airflow limitation, and which are partly due to a TGF- $\beta 1$ driven epithelial reprogramming.

@ERSpublications

The COPD airway epithelium is programmed for mesenchymal transition via a TGF- $\beta 1$ dependent process http://ow.ly/LhIQb

This article has supplementary material available from erj.ersjournals.com.

Received: July 72014 | Accepted after revision: Oct 242014 | First published online: March 052015

Conflict of interest: None declared.

Support statement: C.E. Pierreux is postdoctoral specialist of the Fonds National de la Recherche Scientifique, Belgium (grant FRSM 3.4522.12) and investigator of the institute for Walloon Excellence in Lifesciences and Biotechnology, Belgium (WELBIO CR-2012S-05). This study was in part supported by the Fonds National de la Recherche Scientifique, Belgium (grant FRSM 3.4582.08) and by Fonds Spéciaux de Recherche (FSR 2007 and 2011) of the UCL, Belgium, as well as by the Fondation Horlait-Dapsens of the UCL, Belgium and PHU-RINNOPARI of the CHU of Reims, France.

Copyright OERS 2015 


\section{Introduction}

Chronic obstructive pulmonary disease (COPD) is a frequent chronic disease causing a major health burden and will become the third most common cause of death by 2030 [1]. It is associated with abnormal airway and alveolar responses to noxious exposure, usually to cigarette smoke. Chronic inflammation with activation of neutrophils and macrophages and imbalance between proteinases/ anti-proteinases and oxidants/anti-oxidants have been implicated in this process [2]. In addition, structural changes in COPD are also present and differ between airways and alveoli. In contrast to respiratory bronchioles and alveoli which are disrupted, causing so-called emphysema, matrix deposition and subepithelial fibrosis are present in conducting airways, along epithelial changes. It is likely that airway fibrosis and parenchymal emphysema underlie the irreversible nature of airway obstruction, which is mostly insensitive to current therapies [3].

The airway epithelium assures frontline innate defence mechanisms, through a physical barrier and secretion of protective factors. The barrier function is provided by apical complexes of tight junctions containing zonula occludens-1 (ZO-1) protein that binds occludin and adherens junctions with $\beta$-catenin that binds transmembrane E-cadherin. In addition, ZO- 1 and $\beta$-catenin bind to the actin cytoskeleton [4]. The phenotype of the adult human respiratory epithelium remains "flexible", this plasticity being required to maintain epithelial integrity following injury. Normal airway epithelial repair involves the rapid de-differentiation of airways epithelial cells to produce squamous cells (metaplasia), which is reversible and mediates restitution of the normal airway [5]. The epithelium is then repopulated via resident basal cells, which proliferate and differentiate to form a new epithelium [6]. In addition, airway epithelial cells may dedifferentiate through so-called epithelial-to-mesenchymal transition (EMT), to become able to migrate and to secrete matrix proteins, before differentiating into ciliated or goblet cells. Epithelial cells typically lose, transiently, their epithelial characteristics, with loss of polarity and junctional proteins (such as ZO-1 and E-cadherin), and acquire mesenchymal features such as spindle shape, vimentin filaments and secretion of matrix fibronectin $[5,7]$.

The airway fibrosis observed in COPD is mainly peribronchial, within the adventitia of small airways. In contrast, in asthma, the prominent deposition of extracellular matrix just beneath the epithelial basement membrane occurs in the lamina propria of both large and small airways [8]. In addition, several changes of the bronchial epithelium have been reported in COPD; these include goblet cell hyperplasia and squamous cell metaplasia [9]. EMT participates in normal lung biology during development (airway branching) and repair, but is also observed during cancer progression and metastasis [10]. While alveolar EMT has been reported in lung fibrosis [11], three recent studies suggest that EMT also occurs in COPD airways [12-14]. However, the underlying mechanisms and the functional consequences of EMT in the conducting airways from COPD patients remain unclear.

The present study was therefore designed to assess EMT features of the COPD airway epithelium, both in tissue and in broncho-epithelial cultures differentiated upon air-liquid interface (ALI), hypothesising that EMT in conducting airways from COPD represents an intrinsic feature of the diseased epithelium which contributes to peribronchial fibrosis and thereby, to irreversible airway obstruction. Some of the results of this study have been reported in the form of an abstract at the ERS International Congress 2013 and at the Lung Science Conference 2014 [15, 16].

\section{Materials and methods}

Additional details are provided in the online supplementary material.

\section{Study subjects}

104 patients were enrolled in this study, consisting of 42 controls (26 smokers or ex-smokers, and 16 nonsmokers) and 62 COPD patients, namely 21 mild (Global Initiative for Chronic Obstructive Lung Disease (GOLD) stage I), 22 moderate (GOLD stage II) and 6 severe COPD (GOLD stage III) undergoing lung resection surgery for a solitary tumour and recruited between 2007 and 2013 (table 1). Lung explants from 13 very severe COPD (GOLD stage IV) patients were also included. For all patients, a detailed clinical history was available and all underwent lung function testing. Patients with other lung diseases (such as asthma) were excluded from the study. All patients gave signed informed consent to the study protocol.

\section{Lung tissue sampling and processing}

Lung sections (containing large and small airways) were obtained from the surgical specimens and processed for immunohistochemistry. One additional large airway sample was obtained for primary epithelial cell culture. According to optimal quality of samples, among the 104 enrolled patients (table 1) expression analyses (immunohistochemistry, polymerase chain reaction) were performed for 51 patients 
TABLE 1 Patient characteristics of the study population

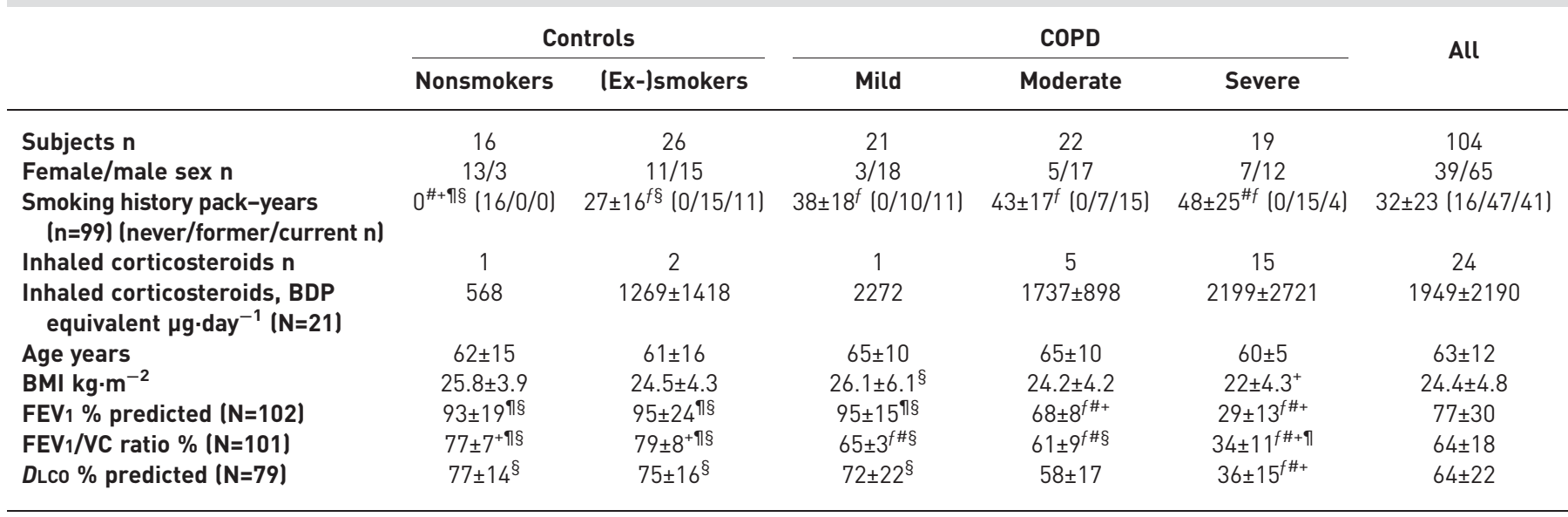

Data are presented as mean \pm SD, unless otherwise stated. Demographic data, lung function tests, smoking history and inhaled corticotherapy are stated for the patient groups, classified according to smoking history and the presence of airflow limitation. Patients with other lung diseases were excluded from the study. $\mathrm{N}$ is specified when data are missing. BDP: beclomethasone diproprionate; BMI: body mass index; FEV1: forced expiratory volume in $1 \mathrm{~s}$; VC: vital capacity; DLCO: diffusing capacity of the lung for CO. \# $: p<0.05$ versus lex-lsmokers; ${ }^{+}: p<0.05$ versus mild COPD; ${ }^{\eta}: \mathrm{p}<0.05$ versus moderate $\mathrm{COPD}^{\S}{ }^{\S}$ : $\mathrm{p}<0.05$ versus severe $\mathrm{COPD}{ }^{f}: \mathrm{p}<0.05$ versus nonsmokers.

(table E1 in the online supplementary material) and primary broncho-epithelial cultures were derived from 69 patients (table E2 in the online supplementary material). In addition, small conducting airways (membranous bronchioles, diameter $\leqslant 2 \mathrm{~mm}$ ) were sampled for immunolocalisation in whole tissue by multiphoton microscopy.

\section{Primary cultures of human bronchial epithelial cells}

A piece of large, cartilaginous bronchus away from the tumour site was selected to derive human broncho-epithelial cells (HBEC). Cultures were carried out in air/liquid interface (ALI) for 2 weeks, to allow re-differentiation into a pseudo-stratified, mucociliary airway epithelium [5]. Cytospins were performed both before starting culture and at 10 days of culture, in submerged conditions. For blocking experiments, anti-human transforming growth factor (TGF)- $\beta 1$ antibody or control mouse immunoglobulin (Ig)G was added (every other day with fresh medium) during the 2 weeks of ALI. No significant cytotoxicity was observed (release of lactate dehydrogenase $<10 \%$ ) in the presented conditions. For kinetics experiments, ALI cultures were carried out for 1-5 weeks and compared with submerged cultures.

\section{Immunoassays for epithelial/mesenchymal markers}

Immunophenotyping for epithelial and mesenchymal markers

Serial paraffin sections of lung tissue and of HBEC filters were stained for junctional proteins (ZO-1, E-cadherin), epithelial lineage markers (p63, $\beta$-tubulin IV, MUC5AC) and vimentin. Quantification of the staining intensity was carried out in 10 fields per section by using ImageJ software (National Institutes of Health, Bethesda, MD, USA), and results were expressed as the percentage of positive/stained area within the epithelium for ZO-1 and E-cadherin. Counting of vimentin-positive cells was performed manually, considering positive columnar cells and excluding intraepithelial leukocytes which also express vimentin (CD45 staining; fig. E1 in the online supplementary material). Thickness of the reticular basement membrane (RBM) was assessed according to the Wilson's method [17]. In immunofluorescence, tissue and HBEC paraffin sections were stained for low molecular weight cytokeratins and vimentin.

\section{Western blot for epithelial/mesenchymal markers}

HBEC were assayed for E-cadherin, vimentin, ZO-1, low molecular weight cytokeratin and fibronectin expression by western blot and quantification was carried out by using Quantity One software (Bio-Rad Laboratories, Hercules, CA, USA). 
Real-time quantitative polymerase chain reaction analysis for Z0-1, E-cadherin, and vimentin mRNA

Total RNA was isolated from HBEC and reverse-transcribed. Expression levels of ZO-1, E-cadherin and vimentin, mRNA were quantified by Real-time quantitative polymerase chain reaction (RT-qPCR) and normalised to the geometric mean of housekeeping genes (glyceraldehyde-3-phosphate dehydrogenase and ribosomal protein S18) [18].

\section{Multiphoton imaging of small airways stained for vimentin, laminin and E-cadherin}

Dissected small airways from one control smoker and two severe COPD patients were treated as described [19]. Whole tissue was immunostained for E-cadherin (epithelial marker), laminin (RBM marker) and vimentin (mesenchymal marker). Fluorescent labelling was observed with a LSM510 multiphoton confocal microscope.

\section{Statistical analysis}

Results were expressed as medians and interquartile ranges, unless otherwise stated. A p-value $<0.05$ was considered statistically significant.

\section{Results}

\section{Reduced expression of epithelial markers in the COPD airway epithelium}

In our study population (table 1), we first addressed whether EMT was potentially present in conducting airways of COPD patients, lung sections from COPD versus control patients were immunostained for junctional markers of the epithelium. In the large airway epithelium from COPD patients, ZO-1 expression was decreased in mild and moderate COPD (fig. 1a and b). E-cadherin expression was also decreased in COPD airways, compared with nonsmokers (fig. la and c).

\section{Increased expression of vimentin in the COPD airway epithelium}

Vimentin-expressing epithelial cells were then analysed as key markers of EMT-related de-differentiation. These cells were increased in both large and small airways from COPD patients (fig. 2a-c). In addition, epithelial expression of vimentin correlated with airway obstruction in terms of post-bronchodilator forced expiratory volume in $1 \mathrm{~s}$ (FEV1) and FEV1/vital capacity (VC) ratio, both in large (fig. $2 \mathrm{~d}$ and e) and small airways (fig. $2 \mathrm{f}$ and g). In contrast, E-cadherin expression positively correlated with FEV1/VC ratio ( $r=0.41, \mathrm{p}=0.006$; not shown). Moreover, expression of epithelial and mesenchymal markers was correlated to each other (table E3 in the online supplementary material).

Multiphoton imaging of small airways (bronchioles) immunostained for vimentin, E-cadherin and laminin demonstrated that the microlocalisation of vimentin-expressing columnar epithelial cells was correlated with the accumulation of vimentin in subepithelial tissue (fig. 3a, left and middle panel, video E1 in the online supplementary material). Co-localisation of vimentin and low molecular weight cytokeratin to these intraepithelial cells indicated the epithelial origin of these cells (fig. 3a, right panel). We also found RBM thickening (fig. $3 b$ and c) and increased number of clefts (fig. $3 b$ and d) in the RBM of airways from COPD patients compared with controls. The number of clefts was correlated to RBM thickness ( $r=0.491$; $\mathrm{p}<0.0001$ ). In addition, RBM thickness was correlated with epithelial expression of vimentin (fig. 3e) and with airflow limitation (FEV1) (fig. 3f). Together, these data confirm the presence of EMT in both large and small airways of COPD patients and showed that this epithelial process correlates with peribronchiolar fibrosis and airway obstruction.

EMT features of the bronchial epithelium from COPD patients persist in vitro

In order to evaluate whether EMT could represent an intrinsic feature of the COPD airway epithelium, we used ALI-HBEC as an in vitro model to study the (re)differentiation process (fig. $4 \mathrm{a}$ ). We found that the bronchial epithelium reconstituted from large airway tissue of COPD patients cultured upon ALI for 2 weeks, displays decreased expression of ZO-1 and E-cadherin (fig. 4b-d), as observed in situ. ZO-1 and E-cadherin mRNA showed a trend to decrease in cultures coming from COPD patients without reaching statistical significance (fig. 4e and f). In addition, expression levels of ZO-1 and E-cadherin in ALI-HBEC were correlated with airway obstruction, in terms of $\mathrm{FEV}_{1}$ and $\mathrm{FEV} 1 / \mathrm{VC}$ ratio (fig. $4 \mathrm{~g}$ and fig. E2 in the online supplementary material).

Increased vimentin expression was also recapitulated in the ALI-cultured epithelium from severe COPD (fig. 5a and c). Co-localisation of cytokeratin and vimentin by immunofluorescence allowed us to exclude fibroblast contamination of the epithelium (fig. 5b). In addition, vimentin induction in HBEC from COPD patients was also observed at the mRNA level (fig. 5d) and was negatively correlated with FEV1 and FEV1/ $\mathrm{VC}$ ratio at the protein level (fig. 5e and $\mathrm{f}$ ). The mesenchymal profile of the COPD airway epithelium was confirmed by western blot analysis for epithelial and mesenchymal proteins, clearly showing decreased E-cadherin and increased vimentin and fibronectin expression (fig. 5g). 
a)
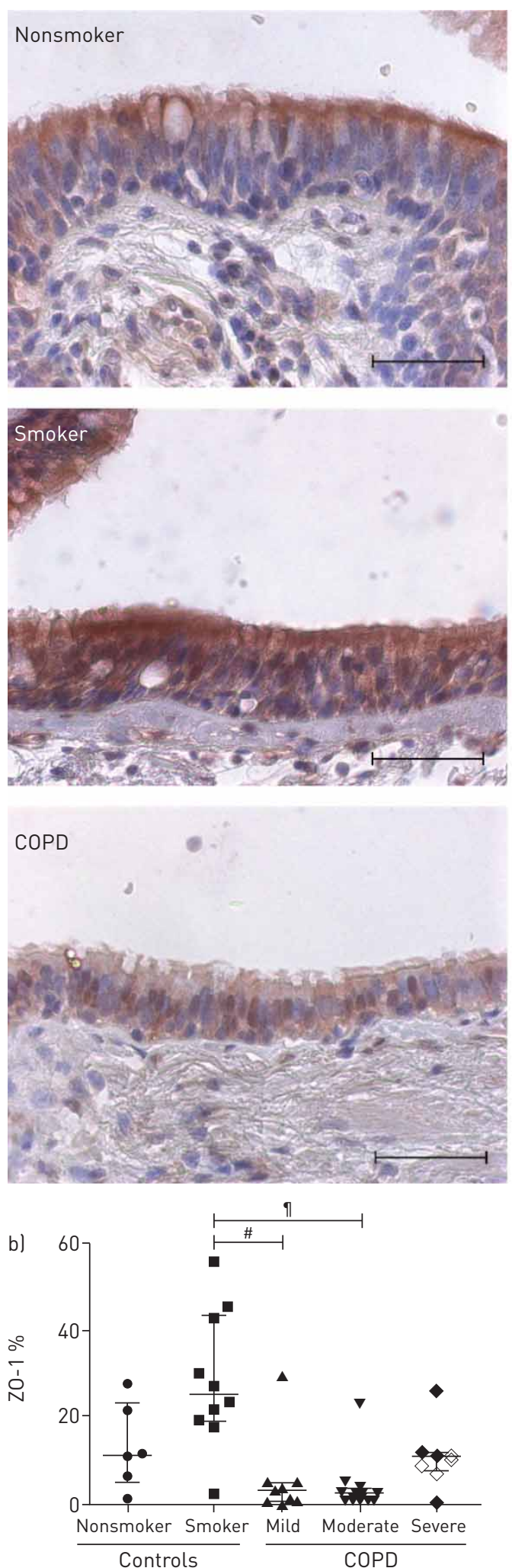

E-cadherin
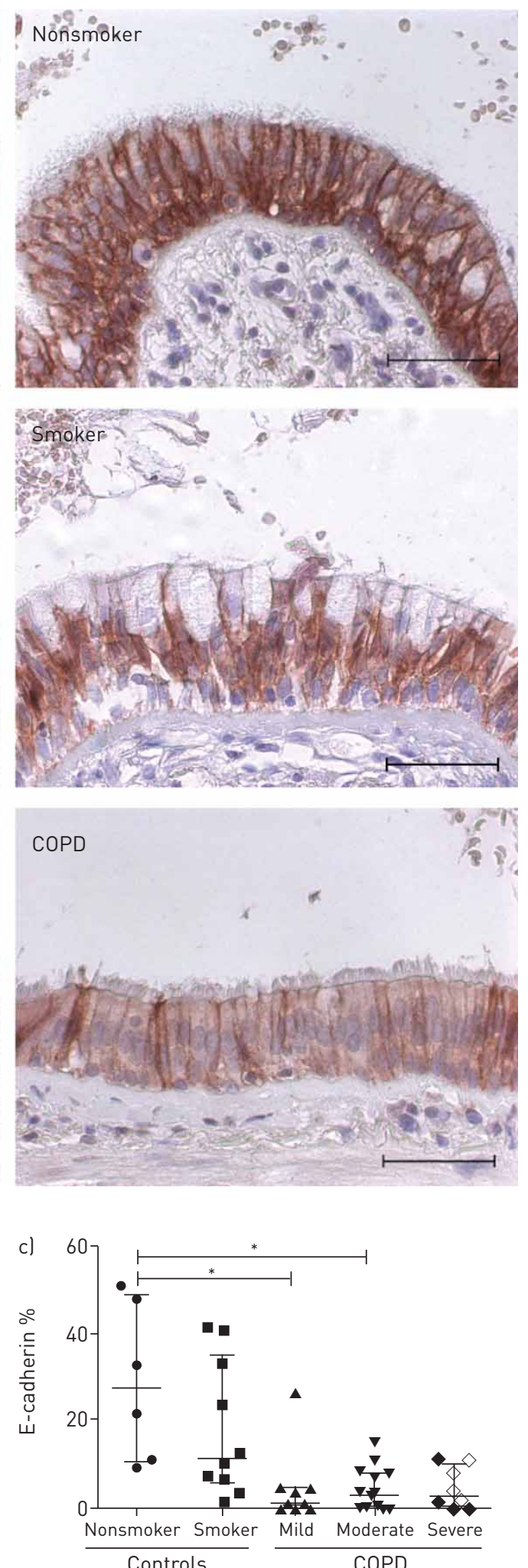

FIGURE 1 Epithelial immunostaining in lung tissue. a) Immunohistochemistry for zonula occludens-1 (ZO-1) and E-cadherin in large airways of a nonsmoker, a smoker and a severe chronic obstructive pulmonary disease (COPD) patient. b) Quantification of $\mathrm{ZO}-1$ staining in large airways ( $\mathrm{n}=47$; empty diamonds represent data from transplanted, Global Initiative for Chronic Obstructive Lung Disease (GOLD) stage IV patients). c) Quantification of E-cadherin staining in large airways $\left(n=47\right.$; empty diamonds represent data from transplanted, GOLD stage IV patients). ${ }^{*}: \mathrm{p}<0.05$; *: $\mathrm{p}=0.006 ;{ }^{\uparrow}: \mathrm{p}=0.001$. Scale bars $=50 \mu \mathrm{m}$. 
a) Large airways
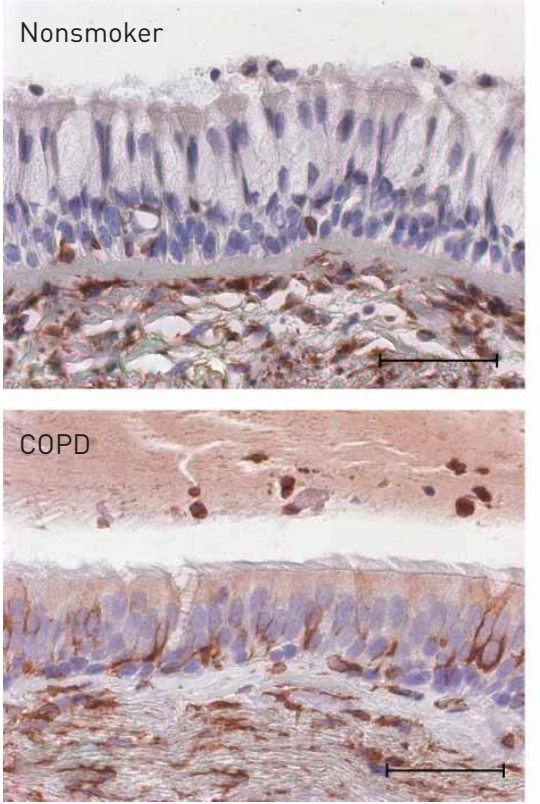

Small airways






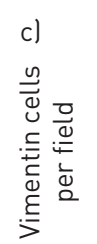

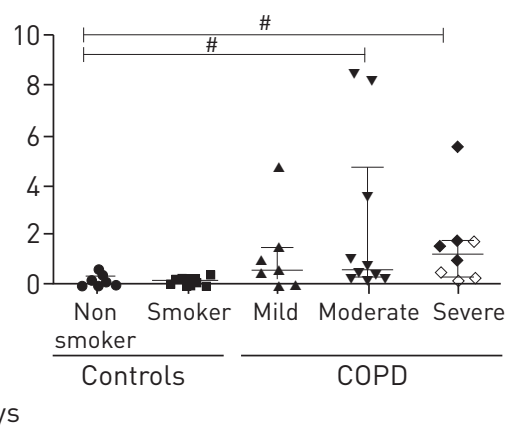

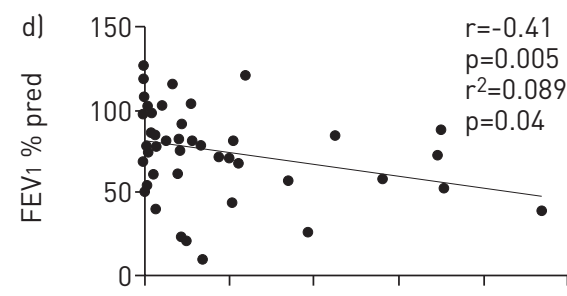

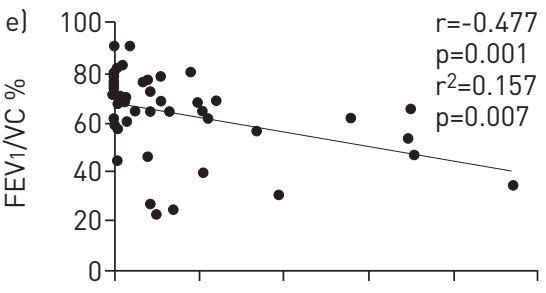

Small airways
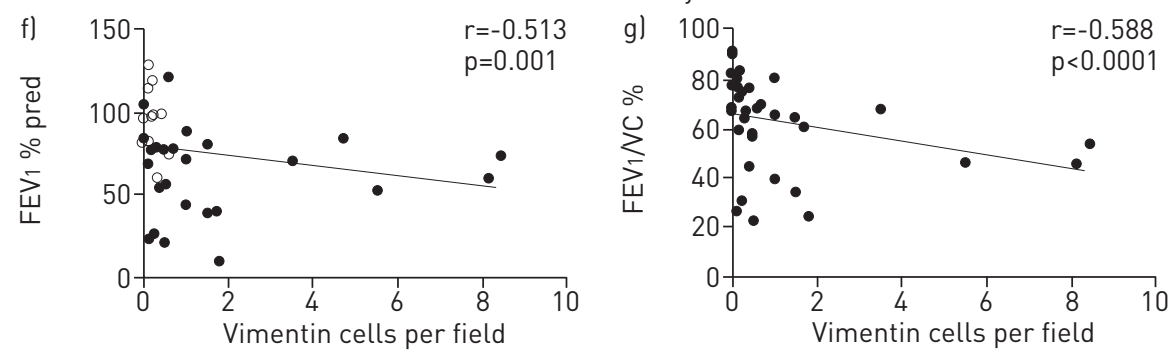

FIGURE 2 Vimentin immunostaining in lung tissue. a) Immunohistochemistry for vimentin in large and small airways of a nonsmoker and a severe chronic obstructive pulmonary disease (COPD) patient. Scale bars $=50 \mu \mathrm{m}$. b) Quantification of vimentin staining in large airways $(\mathrm{n}=48$; empty diamonds represent data from transplanted, Global Initiative for Chronic Obstructive Lung Disease (GOLD) stage IV patients). c) Quantification of vimentin staining in small airways ( $\mathrm{n}=40$; empty diamonds represent data from transplanted, GOLD stage IV patients). d) Correlation between vimentin staining and forced expiratory volume in $1 \mathrm{~s}\left(\mathrm{FEV}_{1}\right)$ in large airways. e) Correlation between vimentin staining and $\mathrm{FEV} 1 /$ vital capacity (VC) ratio in large airways. f) Correlation between vimentin staining and $\mathrm{FEV} 1$ in small airways. g) Correlation between vimentin staining and $\mathrm{FEV} 1 / \mathrm{VC}$ ratio in small airways. *: $\mathrm{p}<0.05$; ${ }^{*}: \mathrm{p}=0.011$; ?: $\mathrm{p}=0.01$. 

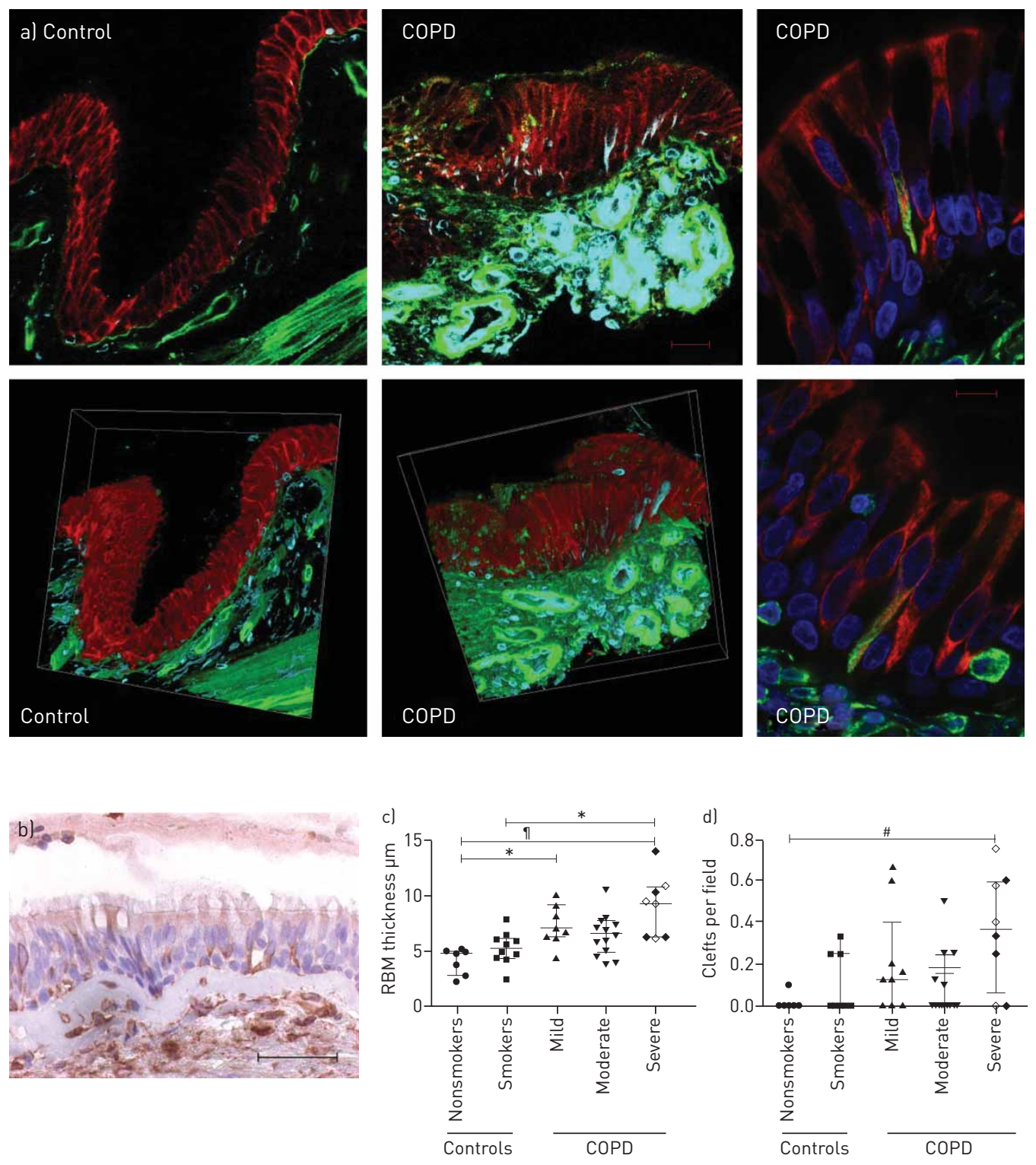

e)

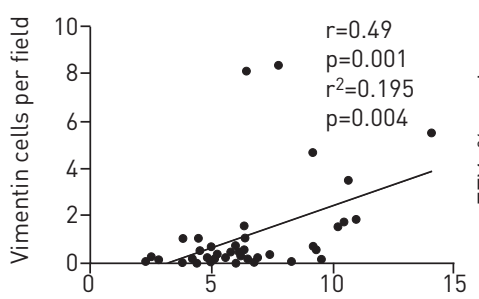

RBM thickness $\mu \mathrm{m}$

f)

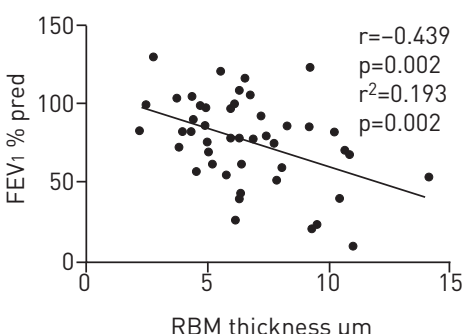

FIGURE 3 Subepithelial fibrosis in chronic obstructive pulmonary disease (COPD). a) Left and middle panels: immunolocalisation of vimentin (in blue), laminin (green) and E-cadherin (red), in a control and a COPD patient. Scale bars $=20 \mu \mathrm{m}$. Right panels: co-immunolocalisation of vimentin (green) and low molecular weight cytokeratin (red), in a goblet and a ciliated cell from a COPD patient. Round, single vimentin-positive cells correspond either to basal cells or intraepithelial leukocytes. Scale bars $=10 \mu \mathrm{m}$. b) Colocalisation of vimentin-positive cells and thickened reticular basement membrane (RBM) in a severe COPD patient. Scale bar=50 $\mu \mathrm{m}$. c) Quantification of RBM thickness in large airways $(n=48$; empty diamonds represent data from transplanted, Global Initiative for Chronic Obstructive Lung Disease (GOLD) stage IV patients). d) Quantification of RBM clefts in large airways ( $\mathrm{n}=48$; empty diamonds represent data from transplanted, GOLD stage IV patients). e) Correlation between RBM thickness and epithelial vimentin staining in small airways. f) Correlation between RBM thickness and forced expiratory volume in $1 \mathrm{~s}(\mathrm{FEV} 1)$. *: p<0.05; \#: $\mathrm{p}=0.03$; ๆ. $\mathrm{p}=0.0011$. 

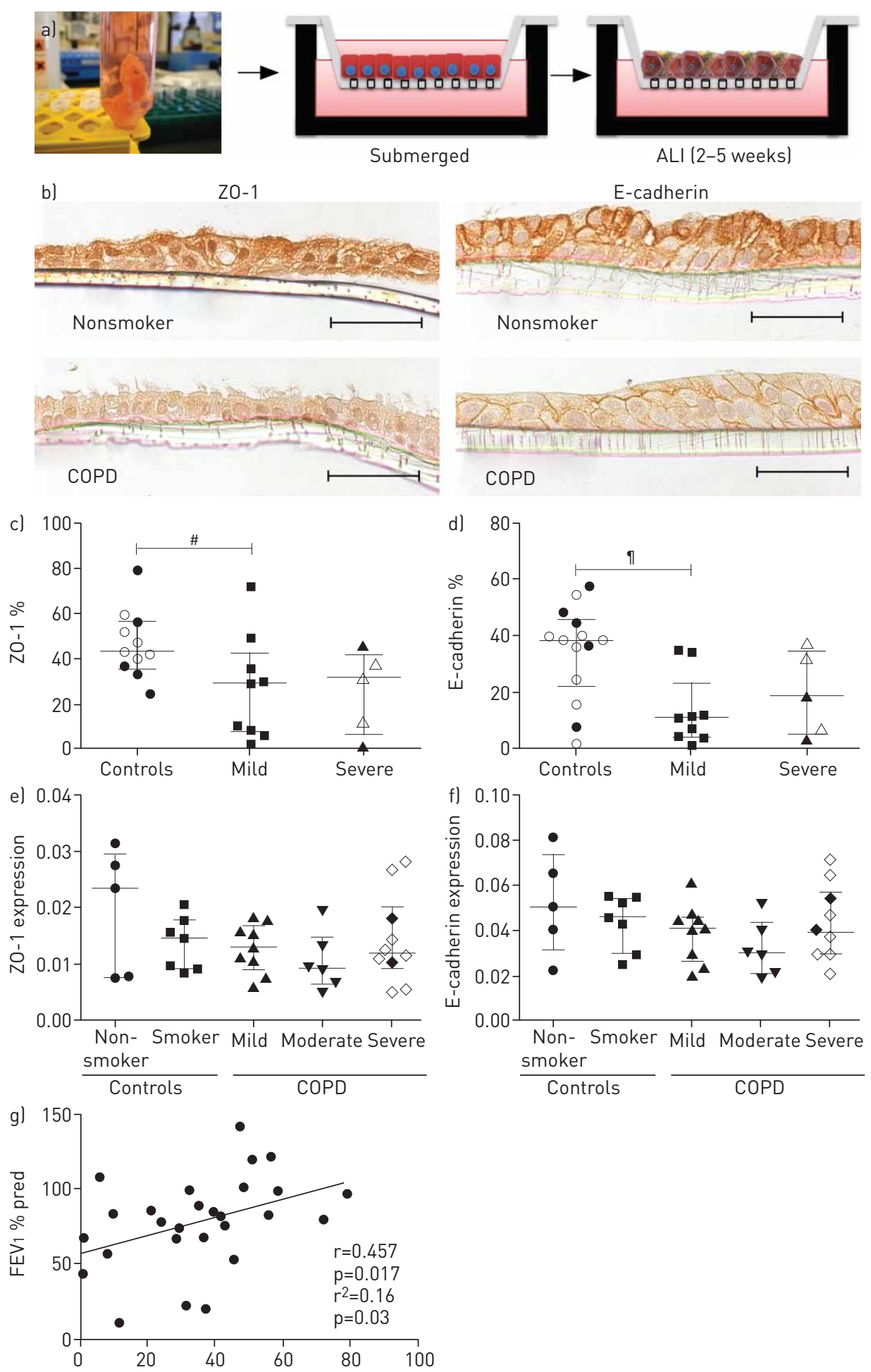

FIGURE 4 Epithelial immunostaining in human broncho-epithelial cells (HBEC) in controls, mild (to moderate) and severe chronic obstructive pulmonary disease (COPD) patients. a) Model of HBEC cultured in air-liquid interface (ALI), from proximal lung tissue. b) Immunohistochemistry for zonula occludens-1 (ZO-1) and E-cadherin in ALI-HBEC from a nonsmoker and a severe COPD patient. Scale bars $=50 \mu \mathrm{m}$. c) Quantification of ZO- 1 staining ( $\mathrm{n}=27$; in controls filled circles represent nonsmokers and, in severe COPD, empty triangles represent data from transplanted, Global Initiative for Chronic Obstructive Lung Disease (GOLD) stage IV patients). d) Quantification of E-cadherin staining ( $n=28$; in controls, filled circles represent nonsmokers and, in severe COPD, empty diamonds represent data from transplanted, GOLD stage IV patients). e) Expression of ZO-1 mRNA by RT-qPCR in ALI-HBEC from controls and COPD patients, normalised to the geometric mean of the two housekeeping genes ( $n=37$; empty diamonds represent data from transplanted, GOLD stage IV patients). f) Expression of E-cadherin mRNA by RT-qPCR in ALI-HBEC from controls and COPD patients, normalised to the geometric mean of the two housekeeping genes ( $\mathrm{n}=37$; empty diamonds represent data from transplanted, GOLD stage IV patients). g) Correlation between ZO- 1 staining and forced expiratory volume in $1 \mathrm{~s}$ (FEV 1 ) values. \#: $\mathrm{p}=0.03$; ${ }^{\uparrow}$ : $\mathrm{p}=0.01$. 
Vimentin

a)

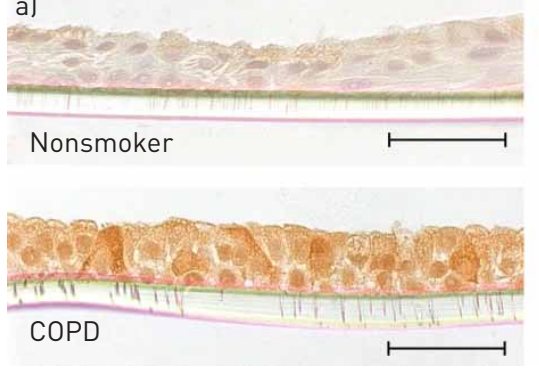


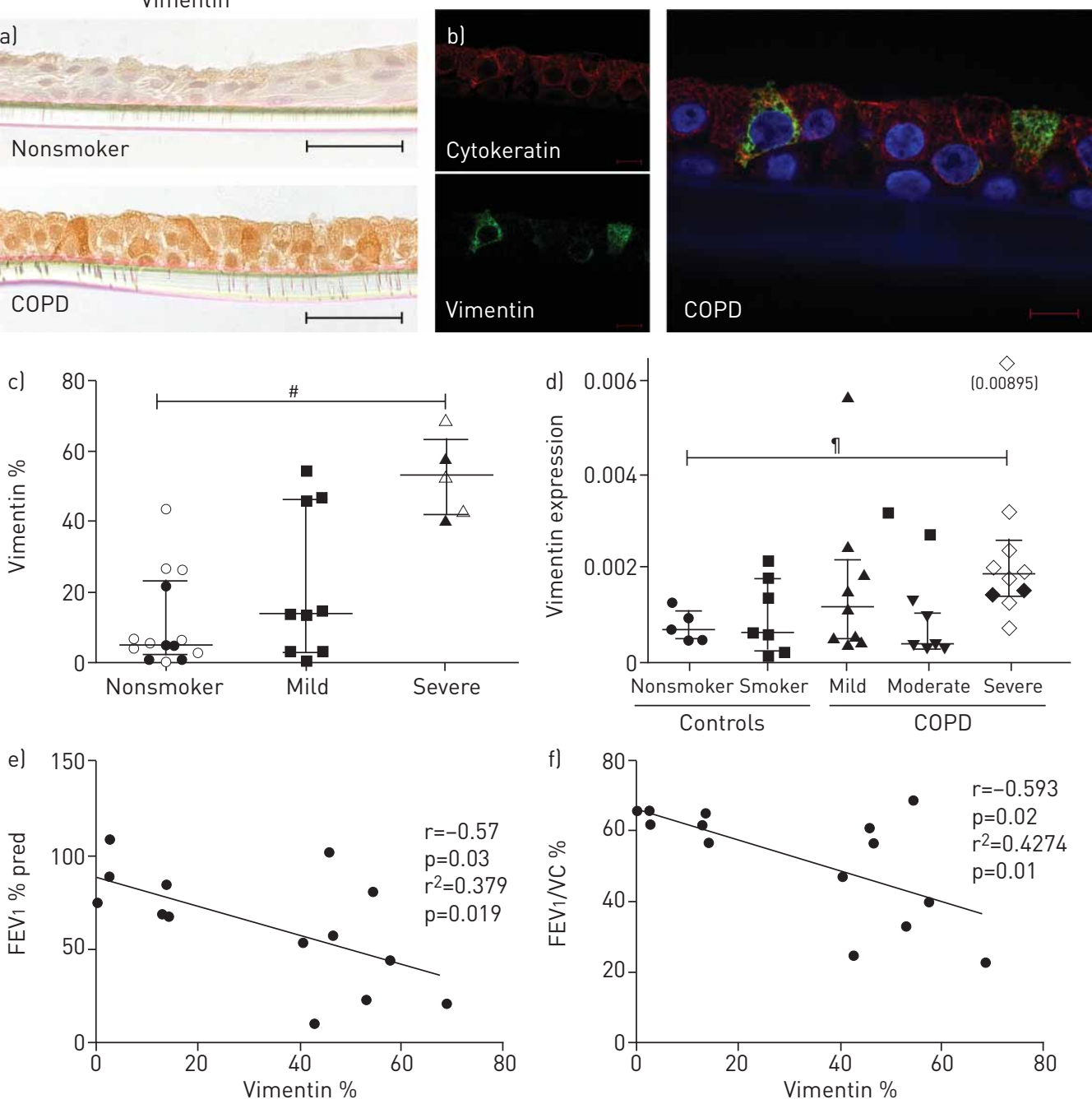

g)

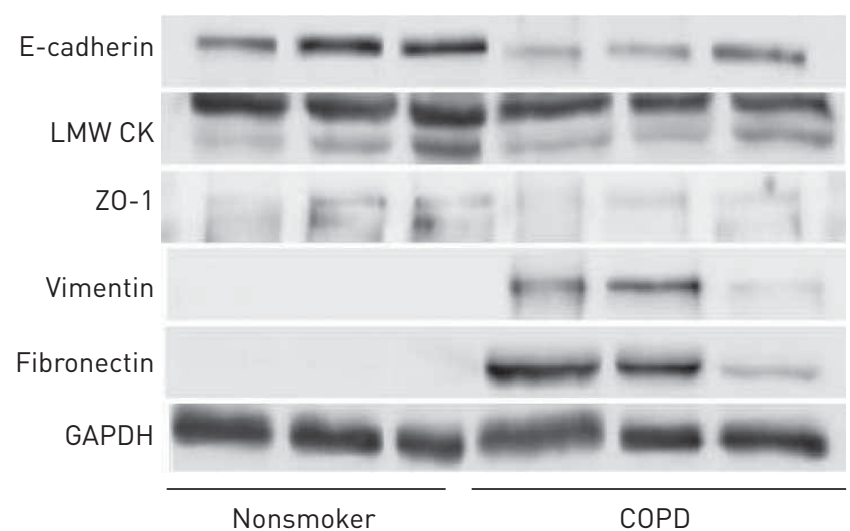

FIGURE 5 Vimentin immunostaining in human broncho-epithelial cells (HBEC) in controls, mild (to moderate) and severe chronic obstructive pulmonary disease (COPD) patients. a) Immunochemistry for vimentin in air-liquid interface (ALI)-HBEC from a nonsmoker and a severe COPD patient. b) Immunofluorescence for co-localisation of vimentin in green and low molecular weight cytokeratin in red in ALI-HBEC from a severe COPD patient. Scale bars=50 $\mu \mathrm{m}$. c) Quantification of vimentin staining $(\mathrm{n}=28$; in controls, filled circles represent nonsmokers and, in severe COPD, empty triangles represent data from transplanted, Global Initiative for Chronic Obstructive Lung Disease (GOLD) stage IV patients). d) Expression of vimentin mRNA, corrected for the geometric mean of the two housekeeping genes ( $\mathrm{n}=37$; empty diamonds represent data from transplanted, GOLD stage IV patients). e) Correlation between vimentin staining and forced expiratory volume in $1 \mathrm{~s}(\mathrm{FEV} 1)$ values in COPD patients $(\mathrm{n}=14) . \mathrm{f})$ Correlation between vimentin staining and $\mathrm{FEV} /$ /vital capacity (VC) ratio in COPD patients $(\mathrm{n}=14)$. g) Immunoblots for epithelial and mesenchymal proteins, as referred to glyceraldehyde-3-phosphate dehydrogenase (GAPDH), in ALI-HBEC from three nonsmokers and three severe COPD patients. LMW CK: low molecular weight cytokeratin; ZO-1: zonula occludens-1. *: $\mathrm{p}=0.006$; " : $\mathrm{p}=0.004$. 
Epithelial ZO-1 and E-cadherin were correlated with each other $\left(\mathrm{r}=0.57, \mathrm{p}=0.002\right.$; and $\left.\mathrm{r}^{2}=0.31, \mathrm{p}=0.003\right)$. When data obtained in lung tissue and HBEC from the same patients $(n=13)$ were compared, significant correlation was observed between in vitro and in situ expression levels of ZO-1 but also between different epithelial junctional proteins ( $\mathrm{ZO}-1$ and E-cadherin in tissue) (table $\mathrm{E} 4$ in the online supplementary material). In addition, E-cadherin expression in HBEC was negatively correlated with vimentin expression in small airway tissue (table E4 in the online supplementary material). Altogether, these data show that the bronchial epithelium reconstituted in vitro from large airway tissue of COPD patients retains EMT features observed in situ, in both large and small airways.

\section{Mesenchymal features of the cultured bronchial epithelium from COPD patients}

Besides vimentin expression, we next evaluated other mesenchymal features of COPD HBEC, namely the morphology and release of matrix proteins. First, HBEC from some COPD patients were morphologically more spindle-shaped cells compared to the classical cobble-stone aspect of epithelial cells from controls (fig. 6a). Secondly, HBEC from some COPD patients (at 2 weeks of ALI) were able to release fibronectin, which was observed with none of the control HBEC (fig. 6b).

\section{Reversibility of EMT features of the cultured COPD bronchial epithelium}

We then wondered whether EMT features observed in the COPD bronchial epithelium (in situ and recapitulated in vitro) related to a reversible reprogramming. To address this issue, the cultured bronchial epithelium was assessed for EMT markers by western blot from the submerged condition to 5 weeks of ALI differentiation (fig. 6c). In the control epithelium, we observed that epithelial markers (E-cadherin and low molecular weight cytokeratins) were upregulated upon ALI, whereas vimentin expression observed in the undifferentiated submerged epithelium was rapidly lost (at 1 week of ALI; fig. 6c), as observed for fibronectin release in the culture medium (fig. 6d). In cultures from COPD patients, epithelial proteins, barely detectable in the submerged conditions, increased upon ALI culture but without reaching the level (particularly for cytokeratins) of the control epithelium. Moreover, expression of the mesenchymal markers vimentin and fibronectin, detected in submerged cultures, persisted up to 2 weeks of ALI before progressively declining. This progressive loss of mesenchymal features was corroborated by fibronectin release data (fig. 6e). Finally, assessing the phenotype of bronchial cells before culturing them and after 10 days of submerged culture showed that vimentin positive cells were present in the originally sampled tissue and thereafter decreased upon culture, before the ALI-driven re-differentiation (fig. 6f).

These results show that EMT features reactivated in vitro upon ALI culture, presumably in cells that are present in the initial biopsy sample, are not persistent and progressively reverse upon the culture conditions.

\section{Contribution of TGF- $\beta$ to EMT reprogramming of the COPD bronchial epithelium}

We next assessed whether TGF- $\beta$, a major inducer of EMT, could contribute to imprint the COPD epithelium for EMT. First, we confirmed that treating the epithelium with exogenous TGF- $\beta 1$ induced vimentin expression, particularly in the basal layer (fig. $7 \mathrm{a}$ and b), as well as fibronectin release (fig. $7 \mathrm{~d}$ ). Conversely, we observed that neutralising TGF- $\beta 1$ (by using a blocking monoclonal antibody) during the 2 weeks of ALI downregulated vimentin expression $(\mathrm{p}=0.03)$, both in control and COPD HBEC, while control mouse IgG had no significant effect (fig. $7 \mathrm{~b}$ ). The morphological appearance changed upon treatment with anti-TGF- $\beta 1$, restoring a cobble-stone shape compared with the spindle-shaped, untreated COPD cells (fig. 7c). In addition, fibronectin release was dose-dependently decreased in ALI-HBEC treated by anti-TGF- $\beta 1$ antibodies (fig. $7 \mathrm{~d}$ and e). Together, these data show that the TGF- $\beta$ pathway is involved in the EMT programming of airway epithelial cells from COPD patients.

\section{Discussion}

This study provides further evidence that EMT occurs in the conducting airways from COPD patients and shows for the first time that de-differentiation and EMT features of the COPD bronchial epithelium correlate with subepithelial fibrosis and airway obstruction. In addition, it also shows that these mesenchymal features are reactivated in vitro, during the first weeks of ALI-driven differentiation of the epithelium reconstituted in vitro from COPD airway tissue, and result at least in part from a reversible reprogramming by TGF- $\beta$.

Dysregulated EMT in the diseased lung was first suggested in parenchymal fibrosis, the alveolar epithelium from patients with idiopathic pulmonary fibrosis showing evidence of EMT and activation of the Wnt/ $\beta$-catenin pathway [20]. It was also shown that EMT could be induced in vitro in (human and rat) alveolar epithelial cells, notably by TGF- $\beta$ [21]. In contrast, the occurrence and relevance of EMT in chronic airway diseases remains debated $[22,23]$. Findings in experimental asthma and in vitro epithelial responses to TGF- $\beta$ and allergens suggested a role for EMT in allergic asthma [24], but direct evidence of constitutive 

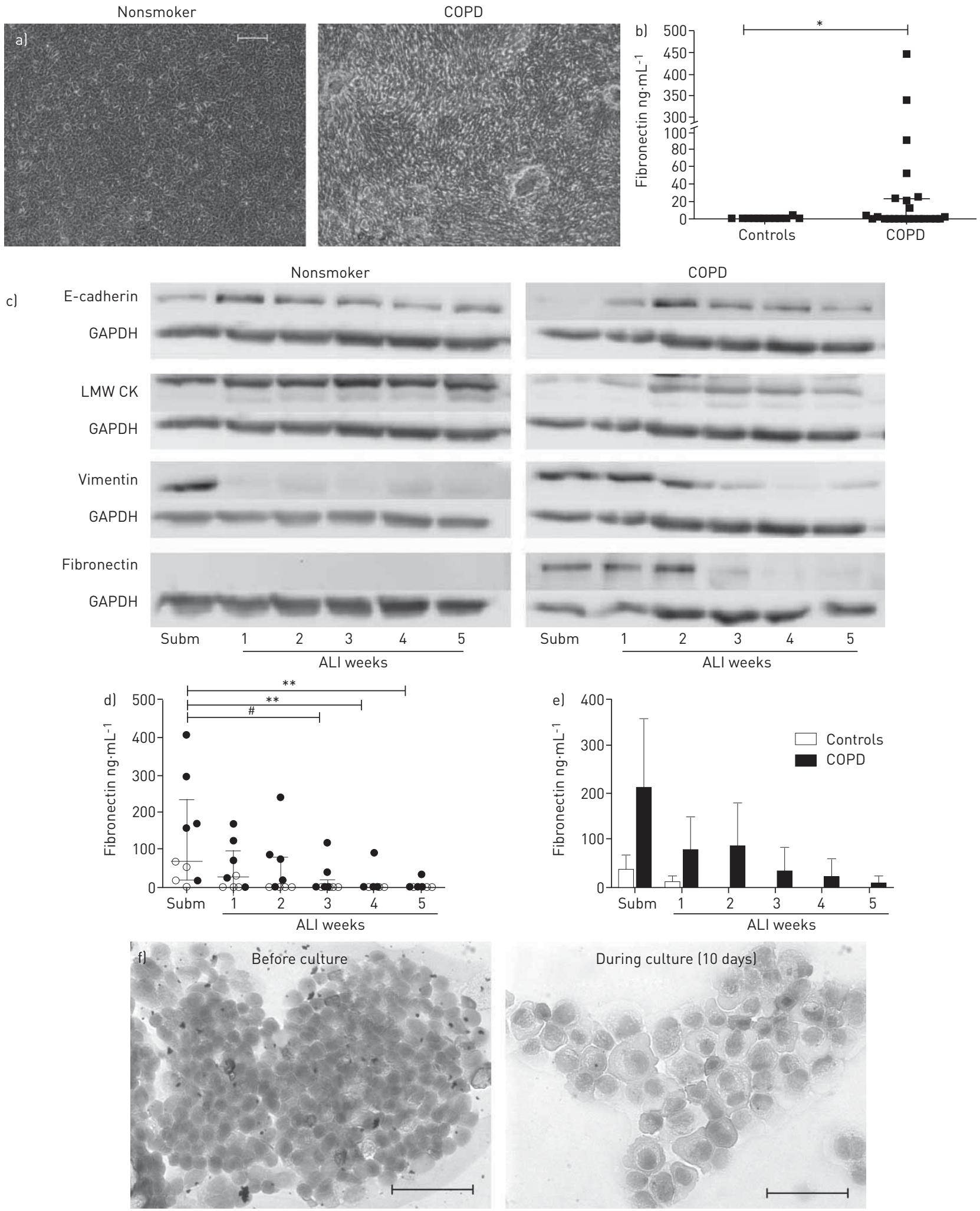

FIGURE 6 Mesenchymal features of human broncho-epithelial cells (HBEC) from chronic obstructive pulmonary disease (COPD) patients. a) Morphological aspect of air-liquid interface (ALI)-HBEC from a control versus a COPD patient. Scale bar $=100 \mu \mathrm{m}$. b) Fibronectin release by HBEC from controls versus COPD, at 2 weeks of ALI $(n=35)$. c) Kinetics of epithelial and mesenchymal markers expression in HBEC, cultured in submerged condition and in ALI (1-5 weeks), with representative Western blots (of $n=7$ experiments) from a control and a COPD patient. d) Fibronectin release by HBEC in submerged and ALI cultures ( $n=9$; filled circles represent COPD patients). e) Fibronectin release data, as grouped for HBEC from COPD versus controls (data are presented as mean \pm SD, $n=9$ ). f) Vimentin immunostaining of a cell suspension from the original airway tissue sample (after digestion and cytospin) from a severe COPD patient, before culture and at 10 days of culture in submerged conditions. Scale bars $=50 \mu \mathrm{m}$. GAPDH: glyceraldehyde-3-phosphate dehydrogenase; LMW CK: low molecular weight cytokeratin; ZO-1: zonula occludens-1. *: $\mathrm{p}<0.05 ;{ }^{*}: \mathrm{p}=0.02 ;{ }^{* *}$ : $\mathrm{p}<0.01$. 

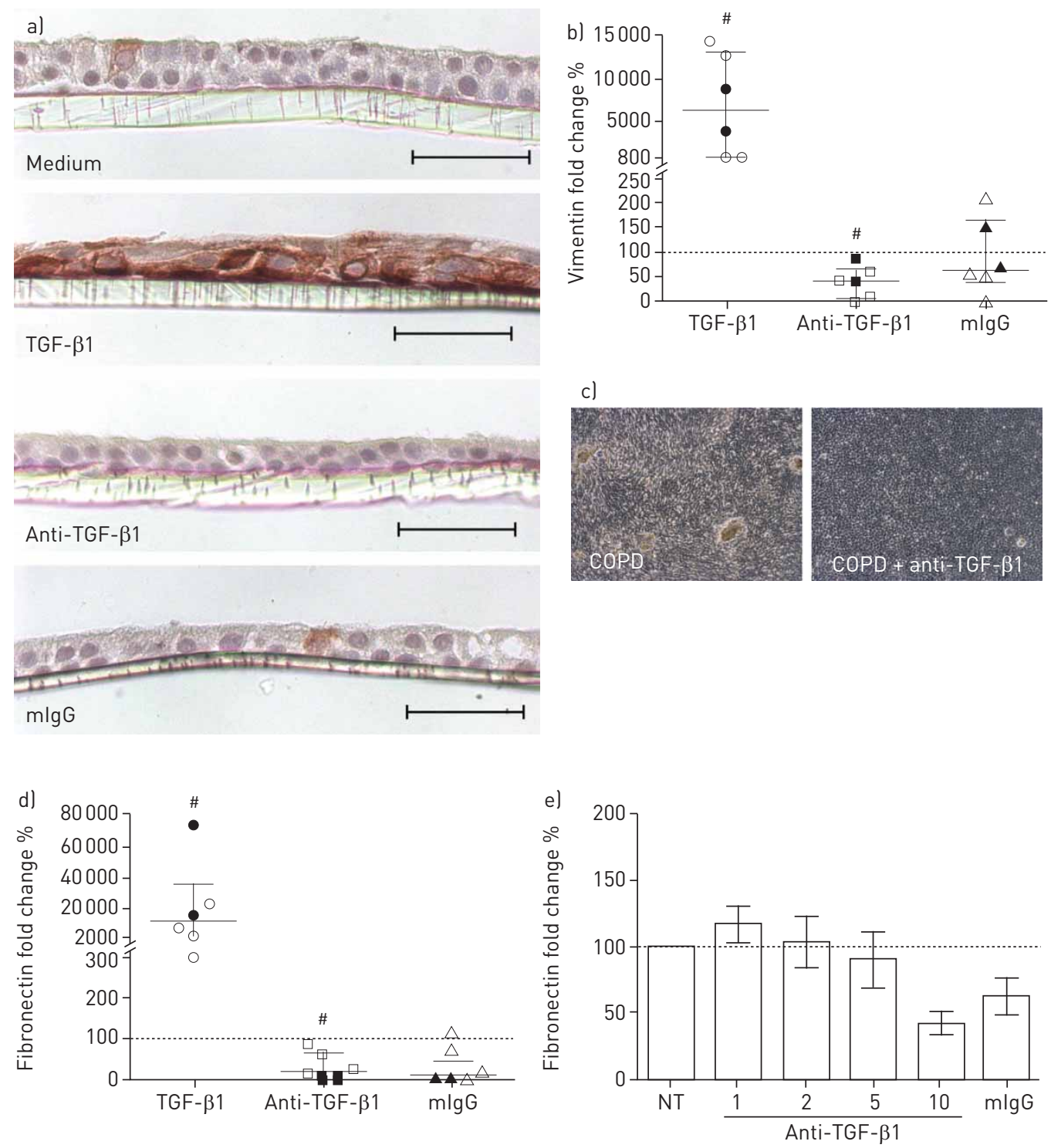

FIGURE 7 Regulation of vimentin and fibronectin expression by transforming growth factor (TGF)- $\beta 1$ and blocking experiments of TGF- $\beta 1$. a) Effect of TGF- $\beta 1\left(10 \mathrm{ng} \cdot \mathrm{mL}^{-1}\right)$, blocking anti-TGF- $\beta 1$ monoclonal antibody $\left(10 \mu \mathrm{g} \cdot \mathrm{mL}^{-1}\right)$, and control mouse IgG $\left(10 \mu \mathrm{g} \cdot \mathrm{mL}^{-1}\right)$ added during the 2 weeks of ALI differentiation on vimentin expression assessed by immunohistochemistry. b) Quantification of vimentin staining ( $\mathrm{n}=6$; filled symbols represent chronic obstructive pulmonary disease (COPD) patients). c) Morphological aspect of air-liquid interface (ALI)-human broncho-epithelial cells (HBEC) with and without anti-TGF- $\beta 1$ antibody. d) Fibronectin release by ALI-HBEC treated by TGF- $\beta 1$, anti-TGF- $\beta 1$ antibody or control mouse immunoglobulin $\mathrm{G}(\mathrm{mIgG})$ during the 2 weeks of ALI differentiation $(\mathrm{n}=6)$. e) Fibronectin release assessed in ALI-HBEC incubated with increasing concentrations of anti-TGF- $\beta 1$ antibody $(n=2$; with each condition in triplicate). ${ }^{*}: \mathrm{p}=0.03$ versus nontreated ALI-HBEC. Scale bars $=50 \mu \mathrm{m}$.

overexpression of mesenchymal markers by the asthmatic bronchial epithelium is lacking. EMT in conducting airways was shown in post-transplant bronchiolitis [25], as well as in chronic rhino-sinusitis [26]. In COPD, SoHAL and colleagues [12,13] observed in large airways from COPD patients the presence of clefts within the RBM containing S100A4/vimentin/ matrix metallopeptidase (MMP)-9 positive epithelial cells, presumably migrating from the surface epithelium to the lamina propria. MiLARA et al. [14] recently reported EMT features in small airways of smokers and COPD patients and in undifferentiated (submerged) primary broncho-epithelial cells from COPD patients. It was shown in vitro that cigarette smoke is also able, per se, to induce EMT $[14,27]$. However, in our data, ZO-1 was not affected in situ, in the airway epithelium from smokers, suggesting that ZO-1 downregulation may only concern susceptible smokers who have developed COPD. Our study of large and small airways from COPD and control patients confirms the presence of de-differentiated epithelial cells engaged in EMT at both levels, suggesting that 
EMT could represent a continuous process along COPD airways. This large series allowed us to correlate findings to lung functional indices of airflow limitation. Interestingly, EMT features of the bronchial epithelium observed in situ (such as vimentin-expressing epithelial cells) correlated with the severity of airway obstruction. In addition, they also correlated with the RBM thickening, which is a salient feature of asthma and also observed in COPD to a lesser extent $[28,29]$. Moreover, by imaging small airway tissue we observed a co-localisation of vimentin-expressing epithelial cells with thickness and fragmentation (clefts) of the RBM and with subepithelial deposition of vimentin. It is therefore proposed that EMT could contribute in COPD to peribronchial fibrosis and subsequently, to irreversible airway obstruction. The fact that changes in ZO-1 and E-cadherin expression were not clearly observed at the mRNA level suggests that they may relate to post-transcriptional regulation, as previously shown for these proteins [30, 31]. This study helps to better understand the pathophysiology of airway fibrosis in COPD through EMT, which was previously implicated in the alveolar epithelium for parenchymal fibrosis or for dysregulated migration during cancer progression. In addition, it remains to study whether dysregulated EMT could represent a common pathway underlying some associated disorders such as mixed emphysema/fibrosis phenotype or the association between COPD and lung cancer.

The "memory" of EMT features in the bronchial epithelium reconstituted in vitro from airway tissue of COPD patients is of particular interest. It has been reported that broncho-epithelial cells from COPD may retain certain aberrant functions, such as for oxidative responses [32], interleukin-8/CXCL8 [33] or MMP-9 production [14]. We show here that broncho-epithelial cells from COPD display de-differentiation/ mesenchymal features which persist up to 2 weeks of ALI culture, and include increased fibronectin release. Interestingly, these phenotypic signatures of the COPD epithelium correlated to some extent with expression data (in situ) and with lung function tests (in vivo), further indicating that this in vitro ALI system represents a valid tool to study epithelial changes in COPD. The possibility that increased numbers of mesenchymal cells within the COPD bronchial epithelium result from the migration of progenitor cells (and not from in situ de-differentiation of epithelial cells) [34], as shown in experimental lung fibrosis [11], was excluded by the observation that these cells co-express epithelial and mesenchymal markers and that EMT was recapitulated in vitro in ALI cultures where retinoic acid counteracts fibroblast growth [35], indicating that the COPD bronchial epithelium is intrinsically abnormal. The contribution of other cells to peribronchial fibrosis, as well as their interactions with epithelial cells, however needs further investigation.

We show that TGF- $\beta 1$ is a regulatory factor that contributes to imprint the COPD epithelium with EMT features. TGF- $\beta$ has been linked to COPD in genetic studies $[36,37]$ and its expression is increased in the COPD airway epithelium, both in large and small airways [38]. First, we confirmed that TGF- $\beta$ promotes EMT in ALI-HBEC, and that this occurs more specifically in basal cells, as previously reported [39]. Interestingly, the abnormal mesenchymal phenotype observed in ALI-HBEC from COPD patients did not persist longer than two weeks of ALI culture. This reversibility may suggest that the in vivo microenvironment is necessary to maintain the mesenchymal phenotype of COPD epithelial cells. It is thus possible that the inflammatory and oxidative environment of COPD airways could condition the aberrant epithelial imprinting by TGF- $\beta$. It is however known that TGF- $\beta$ is induced in the airways of mice exposed to cigarette smoke before inflammation is elicited [40]. In addition, broncho-epithelial cells from COPD patients are primed to release more TGF- $\beta$ [38]. Therefore, it could be speculated that mesenchymal imprinting of the bronchial epithelium by TGF- $\beta$ is an early event in cigarette smoke-induced COPD, which it is maintained on the long term (even after smoking cessation) upon mechanisms that remain to identify. Another hypothesis is that in vitro environment (e.g. retinoic acid), while initially required to promote re-differentiation and related reactivation of abnormal features, finally reverses the epithelial phenotype by affecting epigenetic modifications [41]. Finally, targeting TGF- $\beta 1$ was able to decrease mesenchymal features in vitro, in line with previous studies where anti-TGF- $\beta$ treatment was able to improve both airway pathology and emphysema [42].

In conclusion, this study indicates that EMT-related de-differentiation of the epithelium occurs in COPD conducting airways and correlates with peribronchial fibrosis and with airflow limitation. These changes are recapitulated in vitro, during ALI-driven re-differentiation of the epithelium, at least in part as a consequence of TGF- $\beta$ signalling. These data suggest that EMT is an important component of airway disease in COPD and that its targeting could reveal an attractive therapeutic strategy to restore epithelial barrier and integrity.

\section{Acknowledgements}

The authors thank Pierre Courtoy (de Duve Institute, Université Catholique de Louvain, Brussels, Belgium) and Jérome Ambroise (Université Catholique de Louvain, Brussels, Belgium) for their advice, Alain Poncelet, Valérie Lacroix, Philippe Eucher and Benoît Rondelet (Departments of Thoracic surgery of the Cliniques UCL St-Luc and Department of Thoracic surgery and lung transplantation of CHU Godinne, Belgium, respectively) for their collaboration for tissue sampling, Etienne Marbaix and Jacques Van Snick (de Duve Institute, Université Catholique de Louvain, Brussels, 
Belgium) for help with the tissue biobank and gift of anti-human TGF- $\beta$ antibodies, respectively, Claire Kileztky (University of Reims-Champagne-Ardenne, France) for help with RT-qPCR and Céline Bugli (Université Catholique de Louvain, Brussels, Belgium) for help with statistical analyses.

\section{References}

1 WHO. Global burden disease. www.who.int/healthinfo/global_burden_disease/projections/en/. Date last updated July 2013. Date last accessed: January 2015.

2 Brusselle GG, Joos GF, Bracke KR. New insights into the immunology of chronic obstructive pulmonary disease. Lancet 2011; 378: 1015-1026.

3 Barnes PJ. New anti-inflammatory targets for chronic obstructive pulmonary disease. Nat Rev Drug Discov 2013; 12: 543-559.

4 Valenta T, Hausmann G, Basler K. The many faces and functions of beta-catenin. EMBO J 2012; 31: 2714-2736.

5 Puchelle E, Zahm JM, Tournier JM, et al. Airway epithelial repair, regeneration, and remodeling after injury in chronic obstructive pulmonary disease. Proc Am Thorac Soc 2006; 3: 726-733.

6 Rennard SI. Inflammation and repair processes in chronic obstructive pulmonary disease. Am J Respir Crit Care Med 1999; 160: S12-S16.

7 Xu J, Lamouille S, Derynck R. TGF-beta-induced epithelial to mesenchymal transition. Cell Res 2009; 19: 156-172.

8 Jeffery PK. Remodeling and inflammation of bronchi in asthma and chronic obstructive pulmonary disease. Proc Am Thorac Soc 2004; 1: 176-183.

9 Kim V, Criner GJ. Chronic bronchitis and chronic obstructive pulmonary disease. Am J Respir Crit Care Med 2013; 187: 228-237.

10 Kalluri R, Neilson EG. Epithelial-mesenchymal transition and its implications for fibrosis. J Clin Invest 2003; 112: 1776-1784.

11 Kim KK, Kugler MC, Wolters PJ, et al. Alveolar epithelial cell mesenchymal transition develops in vivo during pulmonary fibrosis and is regulated by the extracellular matrix. Proc Natl Acad Sci USA 2006; 103: 13180-13185.

12 Sohal SS, Reid D, Soltani A, et al. Reticular basement membrane fragmentation and potential epithelial mesenchymal transition is exaggerated in the airways of smokers with chronic obstructive pulmonary disease. Respirology 2010; 15: 930-938.

13 Sohal SS, Reid D, Soltani A, et al. Evaluation of epithelial mesenchymal transition in patients with chronic obstructive pulmonary disease. Respir Res 2011; 12: 130.

14 Milara J, Peiro T, Serrano A, et al. Epithelial to mesenchymal transition is increased in patients with COPD and induced by cigarette smoke. Thorax 2013; 68: 410-420.

15 Gohy S, Detry B, Bouzin C, et al. Increased vimentin expression in human primary bronchial epithelium reconstituted in vitro from patients with COPD. Eur Respir J 2013; 42: Suppl. 57, P3124.

16 Gohy S Detry B, Polette M, et al. Epithelial to mesenchymal transition in the airway epithelium from COPD patients: correlation to disease severity. ERS Lung Science Conference 2014. Available from http://www. ers-education.org/Media/Media.aspx?idMedia $=255936$ Date last updated: September 2014. Date last accessed: November 11, 2014.

17 Wilson JW, Li X. The measurement of reticular basement membrane and submucosal collagen in the asthmatic airway. Clin Exp Allergy 1997; 27: 363-371.

18 Vandesompele J, De Preter K, Pattyn F, et al. Accurate normalization of real-time quantitative RT-PCR data by geometric averaging of multiple internal control genes. Genome Biol 2002; 3: RESEARCH0034.

19 Gaide Chevronnay HP, Janssens V, Van Der Smissen P, et al. Time course of pathogenic and adaptation mechanisms in cystinotic mouse kidneys. J Am Soc Nephrol 2014; 25: 1256-1269.

20 Chilosi M, Poletti V, Zamo A, et al. Aberrant Wnt/beta-catenin pathway activation in idiopathic pulmonary fibrosis. Am J Pathol 2003; 162: 1495-1502.

21 Yao HW, Xie QM, Chen JQ, et al. TGF-beta1 induces alveolar epithelial to mesenchymal transition in vitro. Life Sci 2004; 76: 29-37.

22 Bartis D, Mise N, Mahida RY, et al. Epithelial-mesenchymal transition in lung development and disease: does it exist and is it important? Thorax 2014; 69: p760-765.

23 Pain M, Bermudez O, Lacoste $\mathrm{P}$, et al. Tissue remodelling in chronic bronchial diseases: from the epithelial to mesenchymal phenotype. Eur Respir Rev 2014; 23: 118-130.

24 Johnson JR, Roos A, Berg $\mathrm{T}$, et al. Chronic respiratory aeroallergen exposure in mice induces epithelial-mesenchymal transition in the large airways. PLoS One 2011; 6: e16175.

25 Ward C, Forrest IA, Murphy DM, et al. Phenotype of airway epithelial cells suggests epithelial to mesenchymal cell transition in clinically stable lung transplant recipients. Thorax 2005; 60: 865-871.

26 Hupin C, Gohy S, Bouzin C, et al. Features of mesenchymal transition in the airway epithelium from chronic rhinosinusitis. Allergy 2014; 69: 1540-1549.

27 Heijink IH, Brandenburg SM, Postma DS, et al. Cigarette smoke impairs airway epithelial barrier function and cell-cell contact recovery. Eur Respir J 2012; 39: 419-428.

28 Bourdin A, Neveu D, Vachier I, et al. Specificity of basement membrane thickening in severe asthma. J Allergy Clin Immunol 2007; 119: 1367-1374.

29 Benayoun L, Druilhe A, Dombret MC, et al. Airway structural alterations selectively associated with severe asthma. Am J Respir Crit Care Med 2003; 167: 1360-1368.

30 Noe V, Fingleton B, Jacobs K, et al. Release of an invasion promoter E-cadherin fragment by matrilysin and stromelysin-1. J Cell Sci 2001; 114: 111-118.

31 Coraux C, Kileztky C, Polette M, et al. Airway epithelial integrity is protected by a long-acting $\beta 2$-adrenergic receptor agonist. Am J Respir Cell Mol Biol 2004; 30: 605-612.

32 Pierrou S, Broberg P, O’Donnell RA, et al. Expression of genes involved in oxidative stress responses in airway epithelial cells of smokers with chronic obstructive pulmonary disease. Am J Respir Crit Care Med 2007; 175: 577-586.

33 Comer DM, Kidney JC, Ennis M, et al. Airway epithelial cell apoptosis and inflammation in COPD, smokers and nonsmokers. Eur Respir J 2013; 41: 1058-1067. 
34 Randell SH. Airway epithelial stem cells and the pathophysiology of chronic obstructive pulmonary disease. Proc Am Thorac Soc 2006; 3: 718-725.

35 Daly TJ, Weston WL. Retinoid effects on fibroblast proliferation and collagen synthesis in vitro and on fibrotic disease in vivo. J Am Acad Dermatol 1986; 15: 900-902.

36 Smolonska J, Wijmenga C, Postma DS, et al. Meta-analyses on suspected chronic obstructive pulmonary disease genes: a summary of 20 years' research. Am J Respir Crit Care Med 2009; 180: 618-631.

37 Soler Artigas M, Loth DW, Wain LV, et al. Genome-wide association and large-scale follow up identifies 16 new loci influencing lung function. Nat Genet 2011; 43: 1082-1090.

38 Takizawa $\mathrm{H}$, Tanaka M, Takami K, et al. Increased expression of transforming growth factor-betal in small airway epithelium from tobacco smokers and patients with chronic obstructive pulmonary disease (COPD). Am J Respir Crit Care Med 2001; 163: 1476-1483.

39 Hackett TL, Warner SM, Stefanowicz D, et al. Induction of epithelial-mesenchymal transition in primary airway epithelial cells from patients with asthma by transforming growth factor-betal. Am J Respir Crit Care Med 2009; 180: $122-133$

40 Wang RD, Wright JL, Churg A. Transforming growth factor-betal drives airway remodeling in cigarette smoke-exposed tracheal explants. Am J Respir Cell Mol Biol 2005; 33: 387-393.

41 Kaelin WG, Jr, McKnight SL. Influence of metabolism on epigenetics and disease. Cell 2013; 153: 56-69.

42 Podowski M, Calvi C, Metzger S, et al. Angiotensin receptor blockade attenuates cigarette smoke-induced lung injury and rescues lung architecture in mice. J Clin Invest 2012; 122: 229-240. 